\title{
Exact results for Casimir interactions between dielectric bodies: The weak-coupling or van der Waals Limit
}

\author{
Kimball A. Milton * Prachi Parashar \\ Oklahoma Center for High Energy Physics and Homer L. Dodge Department of Physics and Astronomy, \\ University of Oklahoma, Norman, OK 73019, USA
}

(Dated: October 28, 2018)

\begin{abstract}
In earlier papers we have applied multiple scattering techniques to calculate Casimir forces due to scalar fields between different bodies described by delta function potentials. When the coupling to the potentials became weak, closed-form results were obtained. We simplify this weak-coupling technique and apply it to the case of tenuous dielectric bodies, in which case the method involves the summation of van der Waals (Casimir-Polder) interactions. Once again exact results for finite bodies can be obtained. We present closed formulas describing the interaction between spheres and between cylinders, and between an infinite plate and a retangular slab of finite size. For such a slab, we consider the torque acting on it, and find non-trivial equilibrium points can occur.

PACS numbers: 03.70.+k, 03.65.Nk, 11.80.La, 42.50.Lc
\end{abstract}

The subject of the perturbation of the quantum vacuum energy by material bodies, the so-called Casimir effect, has a long history [1]. For only a limited number of situations, possessing a high degree of symmetry (the interaction energy between infinite parallel planes [2], the self-energy of spheres [3] and cylinders [4]) have exact (numerical) calculations been possible. The experiments carried out during the last decade or so have typically been for a spherical surface above a plane surface. (For a review of the experimental situation, see Ref. [5].) Because the forces for that geometry could not be calculated exactly, comparision with theory was made using the proximity force theorem (PFT), which allows one to compute the force between curved surfaces which are nearly touching [ [6]. However, there is no welldefined "proximity force approximation" that allows one to calculate corrections to the PFT in powers of the ratio of the separation distance to the radius of curvature of the surface. Because the precision of the experiments is now approaching $1 \%$, such corrections may become important.

Various interesting developments have improved the theoretical situation. For example, there has been notable progress in developing the numerical Monte-Carlo worldline method of Gies and Klingmuller 7]. The difficulty with this technique lies in the statistical limitations of Monte Carlo methods and in the complexity of incorporating electromagnetic boundary conditions. Optical path approximations have been studied extensively for many years, with considerable success [8]. However, there always remain uncertainties because of unknown errors in excluding diffractive effects. Direct numerical methods [9], based on finite-difference engineering techniques, may have promise, but the requisite precision of 3-dimensional calculations may prove challenging. A methodology which is, in principle, exact is the multiple scattering formalism, which dates back at least into the 1950s 10, 11, 12, 13]. For more complete references see Ref. 14].

Previous work on this multiple scattering technique, which has been brought to a high state of perfection by Emig et al. [13], has concentrated on numerical results for the Casimir forces between conducting and dielectric bodies such as spheres and cylinders. Recently, we have noticed that the multiple-scattering method can yield exact, closed-form results for bodies that are weakly coupled to the quantum field [14]. This allows an exact assessment of the range of applicability of the PFT. The calculations there, however, as those in recent extensions of our methodology [15], have been restricted to scalar fields with $\delta$-function potentials, so-called semitransparent bodies. (These are closely related to plasma shell models.) Here we remedy that defect by making the extension of the formalism to electromagnetism, and the bodies are, correspondingly, characterized by a permittivity or dielectric constant $\varepsilon$. Strong coupling would mean a perfect metal, $\varepsilon \rightarrow \infty$, while weak coupling means that $\varepsilon$ is close to unity.

In this Letter we will briefly review the formalism, and show that it is precisely equivalent to summing CasimirPolder or van der Waals (vdW) potentials. Exact results have been found in the past in such summations, for example for the self-energy of a dilute dielectric sphere [16] or a dilute dielectric cylinder [17]. Thus it is not surprising that exact results for the interaction of different dilute bodies can be obtained. It is only surprising that such results were not found much earlier. (We note that the additive approximation has been widely used in the past, for example see Ref. [18], but here the method is exact.) We will consider a slab of finite extent above an infinite plane, and calculate the force and torque on the slab. With the center of mass fixed, we find generically the shortest side of the slab aligns with the plate, but for sufficiently square slabs nontrivial equilibrium points can 


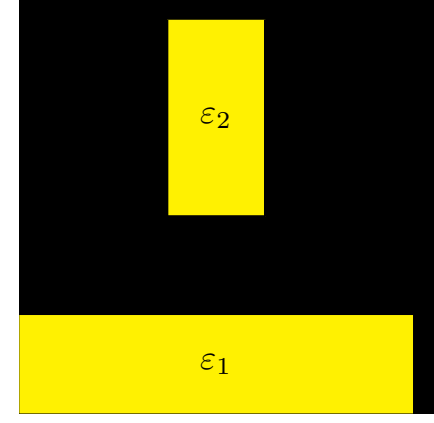

FIG. 1: Slab (with dielectric constant $\varepsilon_{2}$ ) of finite cross section $A$ but of infinite extent in the $z$-direction, a distance $a$ above an infinite plate (with dielectric constant $\varepsilon_{1}$ ) extending to $z=-\infty$.

be found. We will also compute the force between spheres and parallel cylinders. Since the results are exact, we can quantify the deviation from the PFT.

For electromagnetism, we can start from the formalism of Schwinger [19], which is based on the electric Green's dyadic $\boldsymbol{\Gamma}$. Just as in the scalar case, the vacuum energy for a static configuration, existing for a time $\tau$, is given by

$$
E=\frac{i}{2 \tau} \operatorname{Tr} \ln \frac{\boldsymbol{\Gamma}}{\boldsymbol{\Gamma}_{0}},
$$

and again, in precise analogy with the scalar case, the interaction in lowest order between two non-overlapping potentials $V_{1}$ and $V_{2}$ is

$$
E=\frac{i}{2 \tau} \operatorname{Tr} V_{1} \boldsymbol{\Gamma}_{0} V_{2} \boldsymbol{\Gamma}_{0}
$$

Here, the trace is over both vector indices and space-time coordinates, and $\boldsymbol{\Gamma}_{0}$ is the free Green's dyadic.

Now an easy calculation shows that

$$
E=-\frac{23}{(4 \pi)^{3}}\left(\varepsilon_{1}-1\right)\left(\varepsilon_{2}-1\right) \int_{v_{1}}(d \mathbf{r}) \int_{v_{2}}\left(d \mathbf{r}^{\prime}\right) \frac{1}{\left|\mathbf{r}-\mathbf{r}^{\prime}\right|^{7}},
$$

where the bodies, which are presumed to be composed of uniform material filling nonoverlapping volumes $v_{1}$ and $v_{2}$, respectively, are characterized by dielectric constants $\varepsilon_{1}$ and $\varepsilon_{2}$, both nearly unity. This is the famous CasimirPolder potential 20].

Consider first a dilute dielectric slab, a distance $a$ above a dilute dielectric plate filling the half space $z<0$. Let the slab have cross section $A$, and extend from $z=a$ to infinity, as shown in Fig. 1. Because the plate is of infinite extent in the $x-y$ plane, it is easy to carry out the integrals in Eq. (3), with the result being precisely the dilute limit of the Lifshitz formula:

$$
\frac{E}{A}=-\frac{23}{640 \pi^{2}} \frac{1}{3} \frac{\left(\varepsilon_{1}-1\right)\left(\varepsilon_{2}-1\right)}{a^{3}},
$$

from which the force per area is obtained by taking the negative derivative with respect to $a$. There is no correction due to the finite size of the slab. This is presumably a consequence of the fact that for weak coupling, multiple scattering reduces to the two-scattering approximation.

Instead of integrating over $z$ for the slab, we could have considered a slab of thickness $d z$ and area $A$, a distance $z$ above the infinite plate, which has the energy

$$
\frac{d E}{A}=-\frac{23}{640 \pi^{2}}\left(\varepsilon_{1}-1\right)\left(\varepsilon_{2}-1\right) \frac{d z}{z^{4}} .
$$

This allows us to consider an arbitrarily shaped body above the infinite plate. For example, we can immediately find the energy of a dilute sphere of radius $a$, the center of which is a distance $Z$ above the plate, $Z>a$. The energy is

$$
E=-\frac{23}{640 \pi^{2}}\left(\varepsilon_{1}-1\right)\left(\varepsilon_{2}-1\right) \frac{v}{Z^{4}} \frac{1}{\left(1-a^{2} / Z^{2}\right)^{2}},
$$

where $v$ is the volume of the sphere. When the sphere nearly touches the plate, $\delta=Z-a \ll a$, we recover the proximity force theorem:

$$
U=-\frac{23}{640 \pi^{2}}\left(\varepsilon_{1}-1\right)\left(\varepsilon_{2}-1\right) \frac{\pi}{3} \frac{a}{\delta^{2}} .
$$

It is interesting to compare the correction implied by the exact sphere-plate energy (6) to the proximity force theorem result (7)

$$
\frac{E}{U}=\left(1-\nu \frac{\delta}{a}\right), \quad d \ll a,
$$

with $\nu=1$. This is to be contrasted with the results found by Wirzba [10] and by Bordag and Nikolaev [11] for a scalar field with Dirichlet boundary conditions, $\nu=$ $5 / \pi^{2}-1 / 3=0.173$; Maia Neto et al. 12 find for perfectly conducting boundary conditions for electromagnetic field fluctuations $\nu \approx 1.4$. So our dilute dielectrics have an intermediate behavior.

Now, let us return to the slab geometry, but with finite size in all directions, and tilted with respect to the infinite plate. For simplicity, we will consider only a tilt $\theta$ in the $y-z$ plane. See Fig. 2 2 The center of the body is a distance $Z$ above the plane. The result of integrating over the body coordinates is the interaction energy

$$
E=-N \frac{1-\frac{a_{+}(\theta)}{6 Z^{2}}-\frac{1}{3}\left(\frac{a_{-}(\theta)}{4 Z^{2}}\right)^{2}}{\left[1-\frac{a_{+}(\theta)}{2 Z^{2}}+\left(\frac{a_{-}(\theta)}{4 Z^{2}}\right)^{2}\right]^{2}}
$$

where

$$
\begin{aligned}
a_{ \pm}(\theta) & =a^{2} \cos ^{2} \theta \pm b^{2} \sin ^{2} \theta, \\
N & =\frac{23}{640 \pi^{2}}\left(\varepsilon_{1}-1\right)\left(\varepsilon_{2}-1\right) \frac{v}{Z^{4}},
\end{aligned}
$$




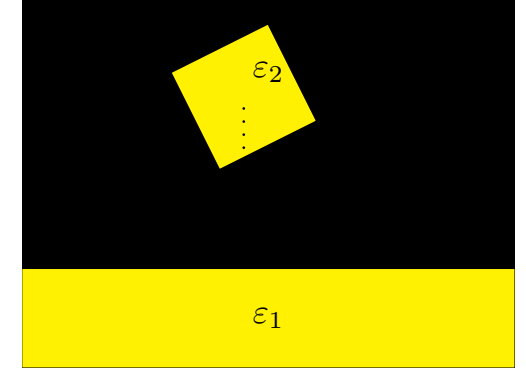

FIG. 2: Rectangular solid (with dielectric constant $\varepsilon_{2}$ ) of side $a, b$, and $c$ (perpendicular to the plane, not shown) a distance $Z$ above an infinite plate (with dielectric constant $\varepsilon_{1}$ ) extending to $z=-\infty$. The side $b$ makes an angle $\theta$ with respect to the plate.

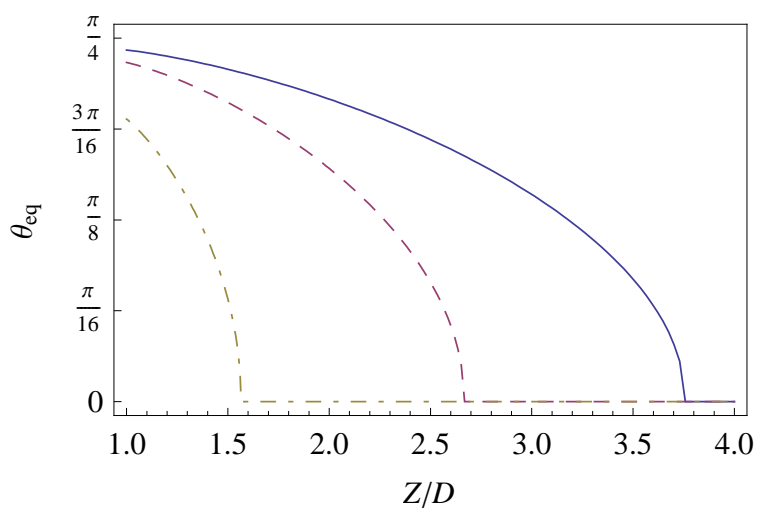

FIG. 3: The stable equilibrium angle $\theta_{\text {eq }}$ of a slab above an infinite plate for given $b / a$ ratios $0.95,0.9$, and 0.7 , respectively given by solid, dashed, and dot-dashed lines. For large enough separation, the shorter side wants to face the plate, but for $Z<Z_{0}$ the equilibrium angle increases, until finally at $Z=D=\sqrt{a^{2}+b^{2}} / 2$ the slab touches the plate at an angle $\theta=\arctan b / a$, that is, the center of mass is just above the point of contact, about which point there is no torque.

and $v$ is the volume of the slab. This always represents an attractive force between the slab and the plate. Most interesting here is the torque exerted about the center of mass of the slab, $\tau=-\partial E / \partial \theta$. Generically, the shorter side wants to align with the plate, which is obvious geometrically, since that (for fixed center of mass position) minimizes the energy. However, if the slab has square cross section, the equilibrium position occurs when a corner is closest to the plate, also obvious geometrically. But if the two sides are close enough in length, a nontrivial equilibrium position between these extremes can occur.

The situation is illustrated nicely in Fig. 3. Consider a slab of aspect ratio $b / a<1$. When one corner of the slab just touches the plate, the center of mass is $Z=D=$ $\sqrt{a^{2}+b^{2}} / 2$ and the equilibrium angle satisfies $\tan \theta=$ $b / a$. For large separation, $Z>Z_{0}$, where

$$
Z_{0}=\frac{a}{2} \sqrt{\frac{2 a^{2}+5 b^{2}+\sqrt{9 a^{4}+20 a^{2} b^{2}+20 b^{4}}}{5\left(a^{2}-b^{2}\right)}}
$$

the stable equilibrium configuration occurs when $\theta=0$, that is, when the shortest face $b$ is closest to the plate. For distances between $Z_{0}$ and $D$, the stable equilibrium angle is intermediate between 0 and $\tan b / a<\pi / 4$, as shown in Fig. 3. For $a=b$ the stable equilibrium position is always at $\theta=\pi / 4$, and for $b=0$ it is at $\theta=0$.

Obviously the method described here can be used to examine interactions between bodies of arbitrary shape. We will content ourselves here with discussing interactions between cylinders and spheres.

First, we remark that it is extremely easy to use this method to reproduce the results given in Ref. [14] for parallel cylinders described by $\delta$-function potentials. It is a bit more complicated to do the calculation for dielectric cylinders, of radii $a$ and $b$, separated by a distance $R>a+b$, because we have to integrate over the radii of the cylinders. With the $1 / r^{7}$ van der Waals potentials, the angular integrals can be carried out explicitly, leaving us with a fairly complicated function of the radial coordinates on each cylinder. To proceed, we expand the integrand of those radial integrals in $r / R, r^{\prime} / R$, and integrate term by term, $\int_{0}^{a} r d r \int_{0}^{b} r^{\prime} d r^{\prime}$. Once more, it is remarkable that we can explicitly carry out the sum:

$$
\frac{E}{L}=-\frac{23}{60 \pi}\left(\varepsilon_{1}-1\right)\left(\varepsilon_{2}-1\right) \frac{a^{2} b^{2}}{R^{6}} \frac{1-\frac{1}{2}\left(\frac{a^{2}+b^{2}}{R^{2}}\right)-\frac{1}{2}\left(\frac{a^{2}-b^{2}}{R^{2}}\right)^{2}}{\left[\left(1-\left(\frac{a+b}{R}\right)^{2}\right)\left(1-\left(\frac{a-b}{R}\right)^{2}\right)\right]^{5 / 2}}
$$

For two spheres, with center-to-center distance $R$ and radii $a$ and $b$ respectively, the calculation is a bit more complex than for cylinders. The reason is that even one angular integration leads to elliptic integrals of complicated argument, so it is difficult to proceed using closedform expressions. Therefore, we expand right at the be- 
ginning, before carrying out any integrations. We then integrate over polar and azimuthal angles, and then over the radial coordinates. We can obtain a power series expansion in powers of $a / R$ and $b / R$, which it is possible to sum, once the power series coefficients are identi- fied. Again we test the procedure by verifying that we reproduce the results found previously for semitransparent spheres [14]. Mathematica is actually able to sum the resulting series, with the following result for the energy

$$
E=-\frac{23}{1920 \pi} \frac{\left(\varepsilon_{1}-1\right)\left(\varepsilon_{2}-1\right)}{R}\left\{\ln \left(\frac{1-\left(\frac{a-b}{R}\right)^{2}}{1-\left(\frac{a+b}{R}\right)^{2}}\right)+\frac{4 a b}{R^{2}} \frac{\frac{a^{6}-a^{4} b^{2}-a^{2} b^{4}+b^{6}}{R^{6}}-\frac{3 a^{4}-14 a^{2} b^{2}+3 b^{4}}{R^{4}}+3 \frac{a^{2}+b^{2}}{R^{2}}-1}{\left[\left(1-\left(\frac{a-b}{R}\right)^{2}\right)\left(1-\left(\frac{a+b}{R}\right)^{2}\right)\right]^{2}}\right\} .
$$

This expression, which is rather ugly, may be verified to yield the proximity force theorem:

$$
E \rightarrow U=-\frac{23}{1920 \pi}\left(\varepsilon_{1}-1\right)\left(\varepsilon_{2}-1\right) \frac{a(R-a)}{R \delta^{2}},
$$

$\delta=R-a-b \ll a, b$. It also, in the limit $b \rightarrow \infty, R \rightarrow \infty$ with $R-b=Z$ held fixed, reduces to the result (6) for the interaction of a sphere with an infinite plate.

In this Letter we have shown that the general methodology of the multiple scattering formulation for weak coupling becomes the pairwise summation of van der Waals energies between the molecules that make up the dilute dielectrics. Such summations have previously been carried out both for the interaction energy between parallel plates [19] and the self-interactions of spheres [16] and cylinders [17], but it was apparently not recognized that it was easy to obtain exact closed-form energies for many interesting situations. Since the calculations here refer to electromagnetic field fluctuations, causing forces and torques on dielectric bodies, these are of far more relevance than our earlier exact results for weak semitransparent bodies. These results raise the intriguing possibility that maybe even in strong coupling, for example, for conducting bodies, exact results may be obtainable. In any case, the results presented here form the basis for a laboratory to study edge effects and other finite-size phenomena which have proved elusive in the past.

We thank the US National Science Foundation (Grant No. PHY-0554926) and the US Department of Energy (Grant No. DE-FG02-04ER41305) for partially funding this research. We thank Archana Anandakrishnan and K. V. Shajesh for extensive collaborative assistance throughout this project. We are particularly appreciative of Steve Fulling's discussions of the "Casimir pistol" that led us to investigate this subject. (See Ref. 21].)

* Electronic address: milton@nhn.ou.edu URL: http://www.nhn.ou.edu/\%7Emilton
† Electronic address: prachi@nhn.ou.edu

‡ Electronic address: wagner@nhn.ou.edu

[1] H. B. G. Casimir, Kon. Ned. Akad. Wetensch. Proc. 51, 793 (1948).

[2] E. M. Lifshitz, Zh. Eksp. Teor. Fiz. 29, 94 (1956).

[3] T. H. Boyer, Phys. Rev. 174, 1764 (1968).

[4] L. L. DeRaad, Jr., and K. A. Milton, Ann. Phys. (N.Y.) 136, 229 (1981).

[5] R. Onofrio, New J. Phys. 8, 237 (2006).

[6] J. Blocki, J. Randrup, W. J. Świątecki, and C. F. Tsang, Ann. Phys. (N.Y.) 105, 427 (1977).

[7] H. Gies and K. Klingmüller, Phys. Rev. D 74, 045002 (2006).

[8] A. Scardicchio and R. L. Jaffe, Nucl. Phys. B 704, 552 (2005). ns, ed. K. A. Milton, (Rinton Press,

[9] A. Rodriguez, M. Ibanescu, D. Iannuzzi, F. Capasso, J. D. Joannopoulos, and S. G. Johnson, Phys. Rev. Lett. 99, 080401 (2007).

[10] A. Wirzba, J. Phys. A 41, 164003 (2008).

[11] M. Bordag and V. Nikolaev, J. Phys. A 41, 164002 (2008).

[12] P. A. Maia Neto, A. Lambrecht, S. Reynaud, arXiv:0803.2444

[13] T. Emig, N. Graham, R. L. Jaffe and M. Kardar, Phys. Rev. Lett. 99, 170403 (2007); Phys. Rev. D 77, 025005 (2008); T. Emig and R. L. Jaffe, J. Phys. A 41, 164001 (2008).

[14] K. A. Milton and J. Wagner, Phys. Rev. D 77, 045005 (2008); J. Phys. A 41, 155402 (2008).

[15] I. Cavero-Peláez, K. A. Milton, P. Parashar and K. V. Shajesh, arXiv:0805.2776 [hep-th]; arXiv:0805.2777 [hep-th]; J. Wagner, K. A. Milton, and P. Parashar, in preparation.

[16] K. A. Milton and Y. J. Ng, Phys. Rev. E 57, 5504 (1998).

[17] K. A. Milton, A. V. Nesterenko and V. V. Nesterenko, Phys. Rev. D 59, 105009 (1999).

[18] M. Bordag, U. Mohideen and V. M. Mostepanenko, Phys. Rept. 353, 1 (2001) arXiv:quant-ph/0106045.

[19] J. Schwinger, L. L. DeRaad, Jr., and K. A. Milton, Ann. Phys. (N.Y.) 115, 1 (1979).

[20] H. B. G. Casimir and D. Polder, Phys. Rev. 73, 360 (1948).

[21] S. A. Fulling, L. Kaplan, K. Kirsten, Z. H. Liu, and K. A. Milton, arXiv:0806.2468. 